\title{
Restriction-ligation-free (RLF) cloning: a high-throughput cloning method by in vivo homologous recombination of PCR products
}

Y. Wang ${ }^{1 *}$, Y. Liu ${ }^{2 *}$, J. Chen ${ }^{3}$, M.J. Tang ${ }^{4}$, S.L. Zhang ${ }^{5}$, L.N. Wei ${ }^{1}$, C.H. Li ${ }^{2}$ and D.B. Wei ${ }^{1}$

${ }^{1}$ Medical College, Qinghai University, Xining, Qinghai, China

${ }^{2}$ Laboratory of Membrane Ion Channels and Medicine,

College of Biomedical Engineering, South-Central University for Nationalities,

Wuhan, Hubei, China

${ }^{3}$ Department of Physiology, School of Medicine, Wuhan University,

Wuhan, Hubei, China

${ }^{4}$ Hunan Provincial Cancer Hospital,

The Affiliated Tumor Hospital of Xiangya Medical School of Central South University,

Changsha, Hunan, China

${ }^{5}$ College of Biology and Food Technology, Anyang Institute of Technology,

Anyang, Henan, China

*These authors contributed equally to this study.

Corresponding authors: C.H. Li / D.B. Wei

E-mail: ionchannels2013@hotmail.com / weidengbang@163.com

Genet. Mol. Res. 14 (4): 12306-12315 (2015)

Received May 25, 2015

Accepted August 6, 2015

Published October 9, 2015

DOI http://dx.doi.org/10.4238/2015.October.9.19

ABSTRACT. In this study, we optimized a restriction-ligation-free (RLF) method to save time and cost of constructing multiple plasmids with the same gene insert, and examined the efficacy of RLF on high-throughput multi-plasmid cloning. This method utilizes the precise DNA repair and recombination systems within Escherichia coli, which allows to bypass the in vitro restriction and ligation enzyme reactions commonly included in 
routine cloning procedures. A homologous arm is linked to the 5'-end of the forward primer used to amplify both the target gene and vector. A different homologous arm is linked to the $5^{\prime}$-end of the reverse primer. Therefore, genes can be cloned into the vectors by homologous recombination after co-transformation of the amplified target gene and the linearized vector, which bear the same homologous arm on either end. More than twentyfour different plasmids were generated by this method, which uses two simple polymerase chain reaction steps. This method is highly efficient in cloning any gene of interest into any vector at any site without sequence constraints, as no restriction and ligation reactions are required.

Key words: In vivo cloning; PCR; High-throughput; Homologous recombination

\section{INTRODUCTION}

Gene cloning, an indispensable technique in molecular biology, is essential for the study of gene expression, regulation, and function. Numerous gene cloning methods, including sticky-end cloning (Scharf et al., 1986), blunt-end cloning (Costa et al., 1994), TA cloning (Holton and Graham, 1991; Marchuk et al., 1991), ligation independent cloning (LIC) (Aslanidis and de Jong, 1990; Shuldiner et al., 1990; Hsiao, 1993; Yang et al., 1993; Kaluz and Flint, 1994; Tillett and Neilan, 1999), and site-specific recombination systems (Hartley et al., 2000), have been developed over the years. These cloning approaches are widely used and have proven to be highly efficacious in most instances; however, they present several limitations because of the extensive enzymatic treatment of the required PCR products or vectors. Furthermore, special and expensive vectors and bacterial strains are required in some cases, such as the In-Fusion Cloning Kit manufactured by Takara Bio Inc. and the Gateway Cloning Kit (Invitrogen). Therefore, novel, streamlined, and cost-effective approaches for gene cloning are the need of the day. Research is currently focused on the development of in vivo cloning techniques (Spiliotis, 2012; Ulrich et al., 2012). These techniques take advantage of the endogenous Escherichia coli enzymatic activities; the PCR products and vectors are modified to incorporate the identical sequences at their respective 5'- and 3'-ends, and are subsequently digested with single or double restriction endonucleases (Spiliotis, 2012). The PCR products and linearized vectors are then simply co-transformed into $E$. coli, thereby bypassing the need for T4-ligase treatment, and greatly simplifying the steps of gene cloning. However, the major impedance to this approach is the difficulty in digesting all plasmids using restriction enzymes, especially when employing double digestions in one solution. The transformation efficiency of residual closed circular DNA is approximately 100-1000 fold (Hanahan, 1983) higher than that of linear DNA (PCR products and linearized vectors). Therefore, a very small amount of uncleaved, closed circular vector plasmid can result in the production of a large amount of unwanted transformants in selective medium. Furthermore, as vectors have different multiple cloning sites (MCS), designing the necessary number of primers with different vector-homologous sequences when cloning multiple genes to different vectors is a tedious and expensive process.

Therefore, a low-cost and high-throughput cloning method is needed to accommodate the high volume of cloning required in systematic molecular biology analyses, such as the analysis of protein-protein interactions by GST pull-down assays, defining protein subcellular localization using fluorescent tracers, and investigating the various cell signaling pathways by manipulating 
the gene expression. To address this need, we have optimized the restriction-ligation-free (RLF) method for vector construction, and examined the efficacy of this approach on high-throughput cloning in multiple vectors. The RLF method utilizes the precise DNA repair and recombination systems in E. coli (Kobayashi and Takahashi, 1988) to bypass the in vitro restriction and ligation enzyme reactions, which are commonly included in routine cloning procedures. RLF cloning allows the seamless cloning of PCR products into multiple PCR-generated vectors. This new approach requires only two PCR steps to amplify the interested genes and vectors, and is free of the limitations presented by vectors, strains, digestion, ligation, and recombinases, and any additional steps (apart from the PCR amplification step).

\section{MATERIAL AND METHODS}

\section{Experiment scheme}

As shown in Figure 1, RLF cloning requires two separate PCR steps to amplify the vector(s) and insert. Short DNA fragments, or homologous arms, were added to both ends of the PCR products. The forward primers for the insert and vector bear the same homologous arm, and reverse primers for the insert and vector share another homologous arm. These homologous arms can be flexibly designed. Following PCR, the insert and vector (flanked by homologous arms) are mixed and co-transformed into E. coli. A typical RLF cloning procedure can be completed in $6 \mathrm{~h}$ (Figure 2). This approach is particularly useful when cloning multiple target genes, as there is no need to custom design different restriction digestions for different vectors.

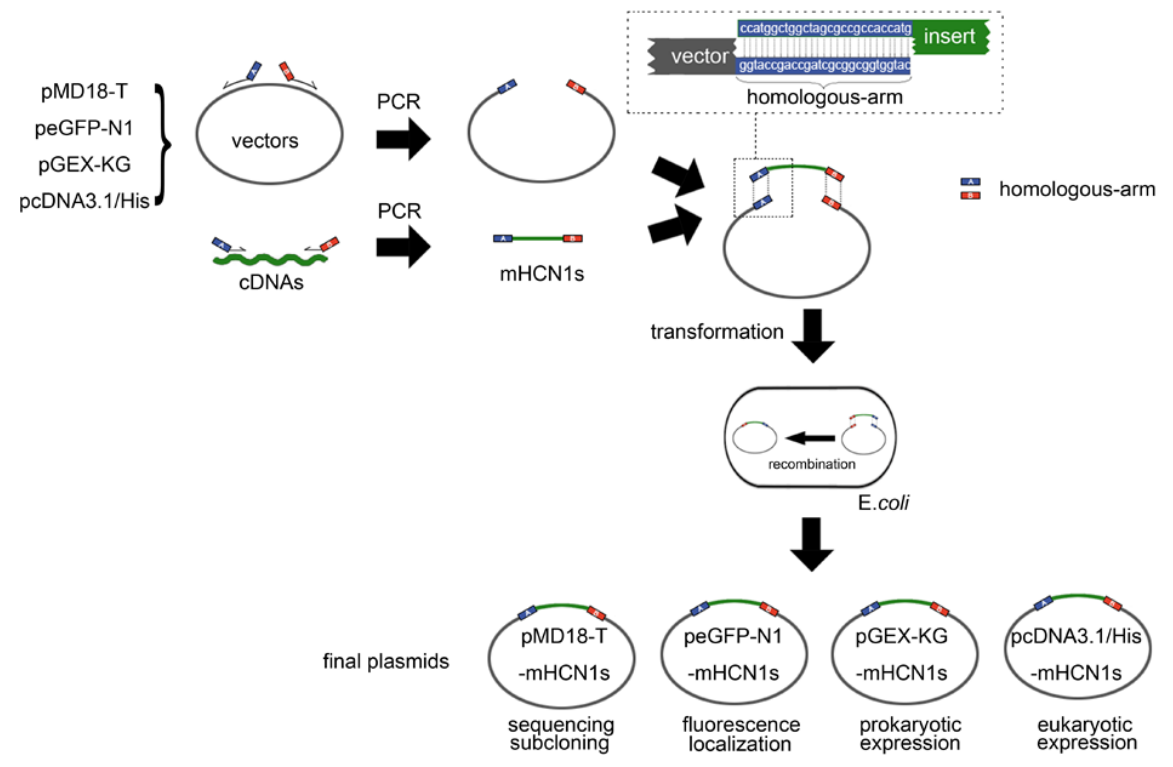

Figure 1. General scheme for the RFL cloning method. PCR products were amplified from vectors and cDNA. Note that the two fragments have the same flanking primer sequences, which were referred to as "homologous arm" sequences. The insert and vector sequences in the homology region hybridize with each other, subsequently forming a partial tetrameric-chain-complex. The vectors used to generate PCR products contain different selectable markers (ampicillin and kanamycin resistance). 
PCR products were amplified from vectors and cDNA. Note that the two fragments have the same flanking primer sequences, which were referred to as "homologous arm" sequences. The insert and vector sequences in the homology region hybridize with each other, subsequently forming a partial tetrameric-chain-complex. The vectors used to generate PCR products contain different selectable markers (ampicillin and kanamycin resistance).

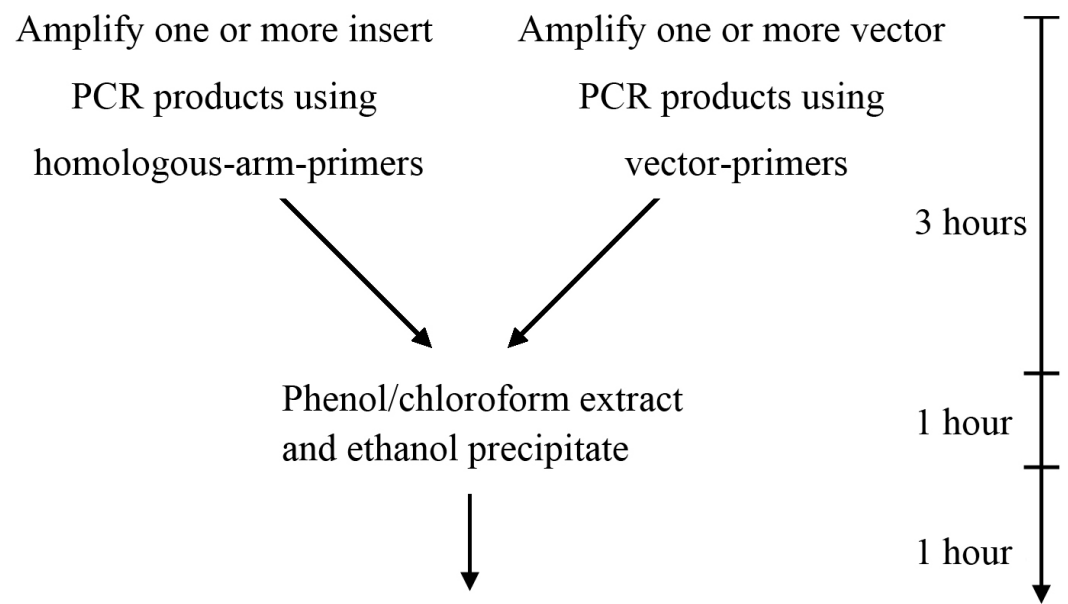

Co-transform into DH5a

Figure 2. Timeline of a typical complete RFL cloning experiment. Several insert genes and vectors can be amplified simultaneously. As this method does not require digestion and ligation by any enzymes, different target genes can be universally cloned into vectors in less than $6 \mathrm{~h}$ utilizing the precise Escherichia coli DNA repair and recombination response.

Several insert genes and vectors can be amplified simultaneously. As this method does not require digestion and ligation by any enzymes, different target genes can be universally cloned into vectors in less than $6 \mathrm{~h}$ utilizing the precise $E$. coli DNA repair and recombination response.

\section{Vectors and primers}

Four vectors, pMD18-T (Takara Bio Inc., Otsu, Japan), peGFP-N1 (Invitrogen, Carlsbad, CA, USA), pGEX-KG (GE Healthcare, Buckinghamshire, UK), and pcDNA3.1/His (Invitrogen), were used in this study. The cDNA of mouse hyperpolarization-activated cyclic nucleotide-gated action channel1 (mHCN1; NM_010408, 2733 bp) was used as the insert template. The primers were synthesized by Genewiz (Genewiz Biotechnology Suzhou, China).

Several primers were designed to generate a series of truncated mHCN1 PCR products (114-2787 bp) to determine whether the insertion size would influence the rate of recombination. All primers consisted of a 27-nucleotide homologous arm at the 5'-end, and a segment complimentary to the template DNA at the 3'-end. All primers utilized in this study are listed in Table 1.

\section{PCR and transformation}

PCR was performed in a $50 \mu \mathrm{L}$ solution containing $0.1 \mathrm{ng}$ vector (template), $200 \mathrm{nM}$ primers, and 2 U Fast PFU DNA polymerase (SinoBio Biotech, Shanghai, China). The PCR was 
run under the following conditions: an initial denaturation step at $94^{\circ} \mathrm{C}$ for $3 \mathrm{~min}$; 35 cycles of denaturation at $94^{\circ} \mathrm{C}$ for $30 \mathrm{~s}$, annealing at $55^{\circ} \mathrm{C}$ for $30 \mathrm{~s}$, and extension at $72^{\circ} \mathrm{C}$ for an appropriate time calculated using the amplification speed of $2 \mathrm{~kb} / \mathrm{min}$; and a final extension at $72^{\circ} \mathrm{C}$ for $5 \mathrm{~min}$. The PCR products were purified by agarose gel electrophoresis and recovered using the DNA Gel Extraction Kit (Axygen, Union City, CA, USA) according to the manufacturer protocols. Purified PCR products were dissolved in TE buffer [10 $\mathrm{mM}$ Tris- $\mathrm{HCl}, 1 \mathrm{mM}$ ethylene diamine tetraacetic acid (EDTA), pH 7.8]. The purity and concentration of total DNA was determined using a ND-2000C spectrophotometer (Thermo Scientific Fisher, Waltham, MA, USA). Two microliters of the vector (700 ng/ $\mu \mathrm{L}, \mathrm{OD} 260 / 280=1.8)$ and $8 \mu \mathrm{L}(1100 \mathrm{ng} / \mu \mathrm{L}, \mathrm{OD} 260 / 280=1.7)$ of the insert were mixed gently and subsequently transformed into chemically competent cells ( $E$. coli XL Gold; Stratagene, La Jolla, CA, USA) prepared by the method described by Inoue et al. (1990) (competency $>10^{9}$ $\mathrm{CFU} / \mu \mathrm{g}$ ). The colonies were screened for the presence of the inserted PCR products by colony PCR. A portion of a bacterial colony was picked using a sterile toothpick. The tip of the toothpick was dipped into $20 \mu \mathrm{L}$ PCR mixture (SinoBio, Biotech, Shanghai, China) containing $100 \mathrm{nM}$ check-primers (Table 1 ). The colony PCR was performed under the following conditions: an initial denaturation step at $94^{\circ} \mathrm{C}$ for $10 \mathrm{~min}$; 35 cycles of denaturation at $94^{\circ} \mathrm{C}$ for $30 \mathrm{~s}$, annealing at an appropriate temperature for $30 \mathrm{~s}$, and extension at $72^{\circ} \mathrm{C}$ for an appropriate period of time; and a final extension at $72^{\circ} \mathrm{C}$ for $5 \mathrm{~min}$. The presence or absence of inserts in the PCR products was assessed by electrophoresis.

Table 1. Forward (F) and reverse (R) primers used in the restriction-ligation-free (RLF) method.

\begin{tabular}{|c|c|c|}
\hline Primer & Sequence* & Length \\
\hline pMD18-T vector (F) & taggatccagcgtagagctgcggatccATCGTCGACCTGCAGGCAT & 46 \\
\hline pMD18-T vector $(\mathrm{R})$ & catggtggcggcgctagccagccatggATCTCTAGAGGATCCCCGG & 46 \\
\hline pGEX-KG vector $(F)$ & taggatccagcgtagagctgcggatccATTCTAGACTCCATGGGTCGA & 48 \\
\hline pGEX-KG vector (R) & catggtggcggcgctagccagccatggCCGGGGATCCACGCGGAACCAG & 49 \\
\hline peGFP vector $(F)$ & taggatccagcgtagagctgcggatccGGCCGCGACTCTAGATCAT & 46 \\
\hline peGFP vector (R) & catggtggcggcgctagccagccatggCTTGTACAGCTCGTCCATG & 46 \\
\hline pcDNA3.1/His vector $(F)$ & taggatccagcgtagagctgcggatcCCACAGTGGCGGCCGCTCGA & 46 \\
\hline pcDNA3.1/His vector (R) & catggtggcggcgctagccagccatggACCCATTTGCTGTCCACCAGTCAT & 51 \\
\hline $\mathrm{mHCN} 1(\mathrm{~F})$ & ccatggctggctagcgccgccaccatggAAGGCGGCGGCAAAC & 43 \\
\hline $\mathrm{mHCN} 1-60(\mathrm{R})$ & ggatccgcagctctacgctggatccTAGACGCTGTTGCCATCGTCGC & 47 \\
\hline $\mathrm{mHCN} 1-120(\mathrm{R})$ & ggatccgcagctctacgctggatccTACGGGGTCCCCAGGCGCTTG & 46 \\
\hline$m H C N 1-240(R)$ & ggatccgcagctctacgctggatccTAATACTGCCGCCGGGGCCCCT & 47 \\
\hline $\mathrm{mHCN} 1-480(\mathrm{R})$ & ggatccgcagctctacgctggatccTACGTCTGCTCTGTGAAGAACGTGAT & 51 \\
\hline $\mathrm{mHCN} 1-960(\mathrm{R})$ & ggatccgcagctctacgctggatccTAAACCCAGCAATCTGGTGGGAAGTC & 51 \\
\hline $\mathrm{mHCN} 1-2733(\mathrm{R})$ & ggatccgcagctctacgctggatccTATAAATTCGAAGCAAAACGGGGT & 49 \\
\hline pMD18-T check (F) & GAGCGGATAACAATTTCACACAGG & 24 \\
\hline pMD18-T check (R) & CGCCAGGGTTTTCCCAGTCACGAC & 24 \\
\hline peGFP-N1 check (F) & GGATCACTCTCGGCATGGACGA & 22 \\
\hline peGFP-N1 check (R) & ССTCTACAAATGTGGTATGGCTGATTATG & 29 \\
\hline pcDNA3.1/His check $(F)$ & TAATACGACTCACTATAGGGAGAC & 24 \\
\hline pcDNA3.1/His check (R) & TAGAAGGCACAGTCGAGGCT & 20 \\
\hline pGEX-KG check (F) & CTGGTTCCGCGTGGATCC & 18 \\
\hline pGEX-KG check (R) & TGAATTAAGCTTGAGCTC & 18 \\
\hline
\end{tabular}

${ }^{*}$ The 27 bp-long homologous arm sequences are denoted in lowercase letters, and the sequence that anneals with the templates are denoted in uppercase letters for all the primer sequences.

\section{Functional validation}

The expression of the inserts was examined by prokaryotic transformation and eukaryotic transfection of the 6 recombinant vectors (mHCN1-60, mHCN1-120, mHCN1-240, mHCN1-480, 
$\mathrm{mHCN} 1-960$, and $\mathrm{mHCN} 1-2733)$, in order to determine if the inserts exhibited normal functionalities in the 4 vectors (pMD18T, pGEX-KG, peGFP-N1, pcDNA3.1/His) created by RLF cloning. The pGEX-KG vector, which contains a fusion glutathione S-transferase (GST-tag) in the 5'-end of the inserts, was transformed into E. coli BL21 (DE3) cells for prokaryotic expression. E. coli BL21 (DE3) cells were cultured in Lysogeny broth media. Plasmid expression was induced by $0.8 \mathrm{mM}$ isopropyl- $\beta$-d-thiogalactopyranoside (IPTG) at $37^{\circ} \mathrm{C}$ for $4 \mathrm{~h}$.

The pEGFP-N1 and pcDNA3.1/His plasmids bearing the inserts were transfected into MCF7 cells to detect the fluorescence localization and eukaryotic expression of the inserts. The cells were transfected as follows: MCF-7 cells were cultured in complete medium for $24 \mathrm{~h}$, and subsequently in antibiotic-free and serum-free RPMI-1640 medium for $8 \mathrm{~h}$. The resuspended cells were then transiently transfected with pEGFP-N1 plasmids $(1 \mu \mathrm{g} / \mathrm{mL}$ per well) using the Turbofect transfection reagent (Hoffmann-LaRoche, Basel, Switzerland). The transfection reagent was removed after 8-9 $\mathrm{h}$, and the cells were cultured in complete medium with antibiotics and serum for $24 \mathrm{~h}$. The protein expression was analyzed by western blotting, as follows: MCF-7 cells with pcDNA3.1/His plasmids (bearing the inserts) were directly lysed with lysis buffer (1\% Triton X-100, $50 \mathrm{mM}$ Tris- $\mathrm{HCl}, \mathrm{pH} 7.4$, $25 \mathrm{mM}$ glycerophosphate, $150 \mathrm{mM} \mathrm{NaCl}, 2 \mathrm{mM}$ EDTA, $2 \mathrm{mM}$ ethylene glycol tetraacetic acid, $1 \mathrm{mM}$ phenylmethylsulfonyl fluoride, $10 \%$ glycerol, and protease and phosphatase inhibitors) for $10 \mathrm{~min}$ on ice. The lysates were collected and centrifuged at $15,000 \mathrm{~g}$ for $15 \mathrm{~min}$ at $4^{\circ} \mathrm{C}$. The supernatant was recovered, and the protein concentration measured using the protein assay kit (Thermo Scientific Pierce, Rockford, IL, USA). The proteins were subsequently separated by sodium dodecyl sulfate polyacrylamide gel electrophoresis (12\% SDS-PAGE), transferred to a polyvinylidene difluoride membrane, blocked in 5\% milk, and incubated with anti-His antibody (Santa Cruz Biotechnology, USA) $(1: 3000)$ at $4^{\circ} \mathrm{C}$ overnight. The blots were incubated with secondary antibody at room temperature for $2 \mathrm{~h}$, and subsequently washed with TBST. Antibody binding was visualized using the electrochemiluminescence kit (Thermo Scientific Pierce, Rockford, IL, USA).

\section{RESULTS AND DISCUSSION}

The influence of the insert size on transformant yield and recombination rates were defined by generating six PCR products containing differentially truncated mHCN1 (114 bp mHCN1-60, 174 bp mHCN1-120, 294 bp mHCN1, 534 bp mHCN1-480, 1014 bp mHCN1-960, and 2787 bp mHCN12733) using one forward primer and a panel of six reverse primers (Figure 3A). Four vectors were amplified by PCR using the vector-primers (Table 1). The amplified pMD18T, pEGPF, and pGEX are displayed in Figure 3B (pcDNA3.1/His is not shown). In order to determine the recombination rates following transformation into XL Gold E. coli, 16 colonies were randomly picked and tested by colony PCR using check-primers (Figure 3C). Transformant yields steadily increased with the decrease in insert size, while the recombination rates remained unaffected by the insert size (Table 2).

E. coli BL21 (DE3) cells harboring pGEX-KG (mHCN1-60, mHCN1-120, mHCN1-240, mHCN1-480, mHCN1-960, or mHCN1-2733) were induced by IPTG, and the expression of the GSTmHCN fusion proteins was subsequently analyzed by SDS-PAGE (Figure 4). The predicted molecular weights of the GST-mHCN recombinant fusion proteins were 28, 31, 35, 44, 61, and $126 \mathrm{kDa}$. The expression yields of mHCN1-120 (31 kDa), mHCN1-240 (35 kDa), mHCN1-480 (44 kDa), and mHCN1-960 (61 kDa) were high because of the properties of the fusion partner (GST-tag). However, mHCN1-2733 (126 kDa) was not expressed in the E. coli cells; this could be attributed to the 37 consecutive glutamines in the $\mathrm{C}$-terminus of $\mathrm{mHCN}$, which does not translate well in $E$. coli cells. 

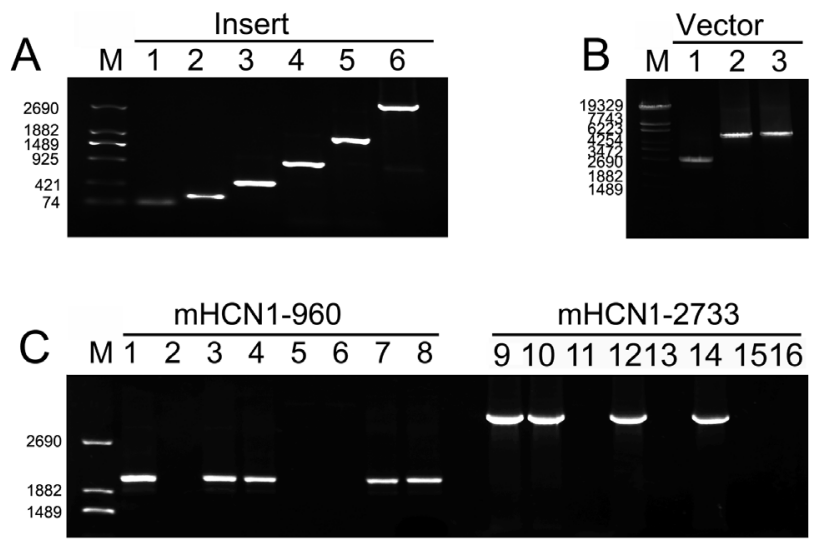

Figure 3. Agarose gel electrophoresis results for the inserts and constructs. A. Six different truncated PCR products of mHCN1. The fragments have a 27-bp homologous arm at both ends. The bands in lanes 1-6 represent the PCR products $(114,174,294,534,1014$, and $2787 \mathrm{bp})$. Lane $M$ indicates the marker. B. PCR products from three different vectors (pMD18-T, peGFP-N1, and pGEX-KG) using vector-primers, which include a 27-bp homologous arm overhang sequence with insert PCR products. Lane $1=$ pMD18-T. Lane $2=$ peGFP-N1. Lane $3=$ pGEX-KG. Lane $M=$ marker. C. Results of the colony PCR screening for positive peGFP-N1 recombinants bearing mHCN1-960 and mHCN1-2733. Lanes 1-8 = colony PCR screening for mHCN1-960-postive colonies. Lanes 9-16 = colony PCR screening for mHCN12733-positive colonies. Lane $M=$ marker.

Table 2. Effect of the vector length and type on the restriction-ligation-free method.

\begin{tabular}{lclllllllc}
\hline Vector & Length $(\mathrm{kb})$ & Usage of vector & Resistance & mHCN1-60 & mHCN1-120 & mHCN1-240 & mHCN1-480 & mHCN1-960 & mHCN1-2733 \\
\hline pMD18-T & 2.7 & Cloning & Amp & $840(81 \%)^{* \Delta}$ & $872(88 \%)$ & $720(69 \%)$ & $648(75 \%)$ & $424(88 \%)$ & $256(81 \%)$ \\
peGFP-N1 & 4.7 & Tracing & Kan & $632(94 \%)$ & $976(88 \%)$ & $392(75 \%)$ & $328(88 \%)$ & $272(69 \%)$ & $288(75 \%)$ \\
pGEX-KG & 5.0 & Expression & Amp & $616(81 \%)$ & $552(88 \%)$ & $496(88 \%)$ & $432(75 \%)$ & $224(69 \%)$ & $112(56 \%)$ \\
pcDNA3.1/His & 5.4 & Expression & Amp & $568(88 \%)$ & $456(75 \%)$ & $472(81 \%)$ & $232(81 \%)$ & $168(88 \%)$ & $56(88 \%)$ \\
\hline
\end{tabular}

${ }^{*}$ Number of total colonies; ${ }^{4}$ fraction of recombinants.

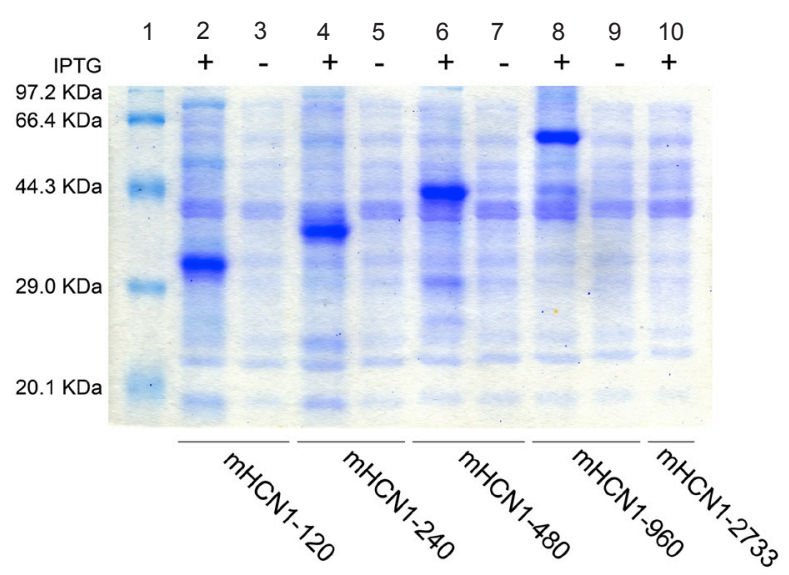

Figure 4. SDS-PAGE was used to identify the protein expression of pGEX-KG-mHCN1-120, $-240,-480$, and -960 , generated by RFL cloning, in prokaryotic cells. Lane 1 = marker; lanes 3, 5, 7, and 9 = before IPTG induction; lanes 2, 4, 6, 8, and 10 = after IPTG induction. 
The fluorescence localization (peGFP-N1) and eukaryotic expression (pcDNA3.1/His) of the recombinant vectors generated by RLF cloning were also analyzed. pEGFP-N1 vectors containing mHCN1-60 or mHCN1-2733 were transfected into MCF-7 cells (Figure 5); these two plasmids, generated by RLF cloning, were successfully expressed in the mammalian cells. Moreover, the His-tagged proteins mHCN1-480 (18 kDa), mHCN1-960 (36 kDa), and mHCN1-2733 $(100 \mathrm{kDa})$ were expressed in cells transfected with recombinant pcDNA3.1/His vectors (detected by blotting; Figure 6). The His-tagged proteins mHCN1-60 (3 kDa), mHCN1-120 (5 kDa), and mHCN1-240 (9 kDa) were not detected because of their low molecular weight. These expression analyses revealed that recombinant vectors generated by RLF cloning are normally expressed in both prokaryotic and eukaryotic cells.

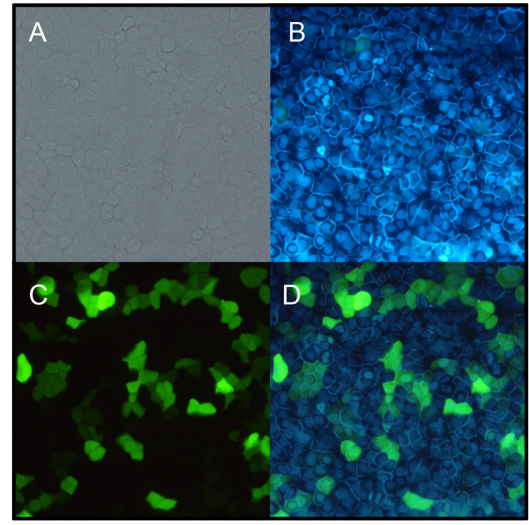

peGFP-N1-mHCN1-60

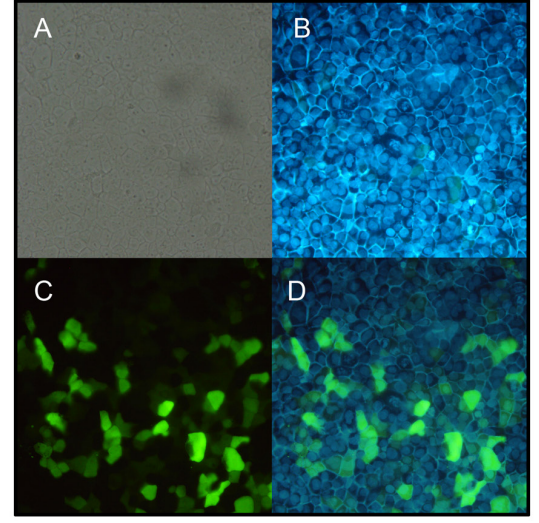

peGFP-N1-mHCN1-2733

Figure 5. Observation of peGFP-N1-mHCN1-60 and mHCN1-2733 expression in MCF-7 cells. The peGFP-N1mHCN1s plasmids generated by RFL cloning are expressed in mammalian cells.peGFP-N1-mHCN1-60 and mHCN12733 presented a comparable transfection efficiency. A. Phase contrast images. B. Images showing GFP expression. C. DAPI images. D. Merged GFP and DAPI images.

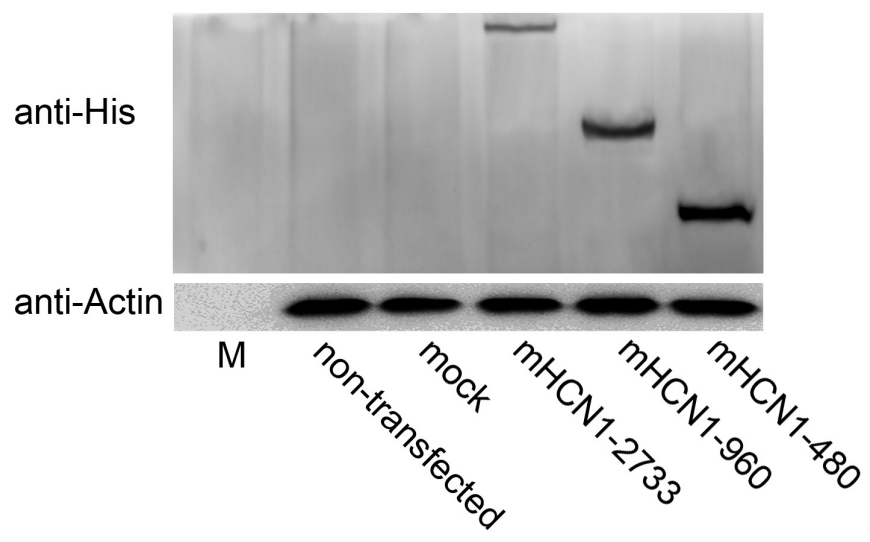

Figure 6. Expression of pcDNA3.1/His-mHCN1-480, -960 and -2733 in MCF-7 cells. The non-transfected and mock groups could not be detected by the anti-His antibody. The mHCN1-480, -960, and -2733-transfected groups presented specific bands. mHCN1-60, -120 , and -240 cannot be detected because of their low molecular weights. 
The RLF cloning approach has several advantages: 1) this approach dispels the need for enzymatic reactions, such as restriction enzyme digestions, alkaline phosphatase treatments, and T4 DNA ligase ligations, thereby reducing the cost and time required for cloning; therefore, this approach is especially suitable for high-throughput cloning experiments. 2) This method is simple, convenient, and rapid because of the minimal requirement of manual handling. 3) This approach enables the direct cloning of the insert into the vectors using an in vivo E. coli homologous recombination system. This is particularly useful in ensuring the correct reading frame of the insert. 4) The vector-primers can be designed for any site on the vector, which allows the insertion of genes of interest into multiple sites in the vector, as well as the modification of the elements in the vectors. 5) The method can be used to introduce mutations, such as deletions and/or insertions, into the target genes using vector-primers with homologous arms located inside the gene. 6) Only 2 homologous arms are used in the primers for multiple vectors and inserts, which minimizes the effort of designing homologous arms.

The selection of appropriate restriction enzymes with cutting sites only in the MCS of the vectors (and not in the inserted genes) is a challenging task, especially when cloning large genes. This is not a concern when using the RLF method, as this approach does not use restriction enzymes. One concern of RLF cloning is the possibility of introducing PCR-derived mutations, which can be minimized by the use of a high-fidelity DNA polymerase for PCRs.

The flexibility afforded by RLF in choosing inserts and vectors, as well as the seamless insertion, easy handling, and the high efficiency of cloning, make this method an ideal protocol for high-throughput recombinant vector construction.

\section{Conflicts of interest}

The authors declare no conflict of interest.

\section{ACKNOWLEDGMENTS}

Research supported by grants provided by the National Natural Science Foundation of China (\#30972848, \#81271234, \#31040011, and \#31260512), the Special Funds of Basic Research Operating Expenses for Universities of China, and funds provided by the Hubei Province Key Laboratory on Cardiovascular, Cerebrovascular, and Metabolic Disorders.

\section{REFERENCES}

Aslanidis C and de Jong PJ (1990). Ligation-independent cloning of PCR products (LIC-PCR). Nucleic Acids Res. 18: 6069-6074. Costa GL, Grafsky A and Weiner MP (1994). Cloning and analysis of PCR-generated DNA fragments. PCR Methods Appl. 3 : 338-345.

Hanahan D (1983). Studies on transformation of Escherichia coli with plasmids. J. Mol. Biol. 166: 557-580.

Hartley JL, Temple GF and Brasch MA (2000). DNA cloning using in vitro site-specific recombination. Genome Res. 10: 1788-1795. Holton TA and Graham MW (1991). A simple and efficient method for direct cloning of PCR products using ddT-tailed vectors. Nucleic Acids Res. 19: 1156.

Hsiao K (1993). Exonuclease III induced ligase-free directional subcloning of PCR products. Nucleic Acids Res. 21: 5528-5529. Inoue H, Nojima H and Okayama H (1990). High efficiency transformation of Escherichia coli with plasmids. Gene 96: 23-28.

Kaluz S and Flint AP (1994). Ligation-independent cloning of PCR products with primers containing nonbase residues. Nucleic Acids Res. 22: 4845.

Kobayashi I and Takahashi N (1988). Double-stranded gap repair of DNA by gene conversion in Escherichia coli. Genetics 119: $751-757$. 
Marchuk D, Drumm M, Saulino A and Collins FS (1991). Construction of T-vectors, a rapid and general system for direct cloning of unmodified PCR products. Nucleic Acids Res. 19: 1154.

Scharf SJ, Horn GT and Erlich HA (1986). Direct cloning and sequence analysis of enzymatically amplified genomic sequences. Science 233: 1076-1078.

Shuldiner AR, Scott LA and Roth J (1990). PCR-induced (ligase-free) subcloning: a rapid reliable method to subclone polymerase chain reaction (PCR) products. Nucleic Acids Res. 18: 1920.

Spiliotis M (2012). Inverse fusion PCR cloning. PLoS One 7: e35407.

Tillett D and Neilan BA (1999). Enzyme-free cloning: a rapid method to clone PCR products independent of vector restriction enzyme sites. Nucleic Acids Res. 27: e26.

Ulrich A, Andersen KR and Schwartz TU (2012). Exponential megapriming PCR (EMP) cloning-seamless DNA insertion into any target plasmid without sequence constraints. PLoS One 7: e53360.

Yang YS, Watson WJ, Tucker PW and Capra JD (1993). Construction of recombinant DNA by exonuclease recession. Nucleic Acids Res. 21: 1889-1893. 